\title{
SALUD MENTAL EN CUIDADORES PRIMARIOS DE NIÑOS CON PARÁLISIS CEREBRAL EN REHABILITACIÓN
}

\section{MENTAL HEALTH IN PRIMARY CAREGIVERS OF CHILDREN WITH CEREBRAL PALSY IN REHABILITATION}

\author{
Rocío Rubio Romero ${ }^{1}$, María del Carmen Gutiérrez Villalvazo ${ }^{1}$ y \\ Amelia Castellanos Valencia ${ }^{2}$ \\ ${ }^{1}$ Psicóloga, ${ }^{2}$ Pediatra. Maestría en Ciencias Médicas \\ Centro de Rehabilitación Infantil Teletón de Occidente, Guadalajara, Jalisco México
}

Cómo referenciar este artículo/How to reference this article:

Rubio Romero, R., Gutiérrez Villalvazo, M.C. y Castellanos Valencia, A. (2015). Salud Mental en Cuidadores Primarios de Niños con Parálisis Cerebral en Rehabilitación. Revista de Psicoterapia, 26(102), 133-144.

\begin{abstract}
Resumen
Objetivo: Identificar la salud mental de los cuidadores primarios de niños con lesión cerebral y su repercusión en la rehabilitación. Método: Se autoadministró a los cuidadores primarios de niños menores de 5 años con lesión cerebral del Centro de Rehabilitación Infantil Teletón Occidente México, el General Health Questionnaire (GHQ-28) validado para detectar morbilidad psicológica y psiquiátrica. Estudio, transversal, analizado con estadística no paramétrica. Resultados: Se incluyeron 131 cuidadores; 77\% femeninos, 23\% masculinos, edad promedio 33 años, rango 20-67. Fueron padres biológicos $95 \%$ de los cuidadores. Los cuidadores mostraron mayor alteración crónica de su salud mental comparada con la aguda 61.8/39.7\%, la disfunción social 45/3\%, la ansiedad/insomnio el primer indicador en un 40.5/12\%, síntomas somáticos 29/7.6\% y depresión grave 9.2/2.3\% ( $p<.001)$. El síntoma con mayor riesgo para ser caso psiquiátrico crónico fue la ansiedad e insomnio crónicos $R M=40.8(p<.001)$. El Retardo mayor de 12 meses en la edad madurativa del niño tuvo asociación con la salud mental aguda RM $3.8(p=.015)$ Los padres con depresión grave presentan elevado número de inasistencias a las terapias de sus hijos ( $p=.05)$. Conclusión: Es importante detectar el riesgo de presentar desórdenes en la salud mental del cuidador primario, debido al efecto negativo directo en el bienestar de ellos $y$ de sus hijos.

Palabras Claves: Ansiedad e insomnio, cuidador primario, disfunción social, parálisis cerebral, salud mental.
\end{abstract}

Fecha de recepción v1: 25/06/2015. Fecha de recepción v2: 6/09/2015. Fecha de aceptación: 30/09/2015. Correspondencia sobre este artículo:

E-mail: rocio_rubio8@hotmail.com

Dirección postal: Centro de Rehabilitación Infantil Teletón de Occidente. Copal 4575 Colonia: Arboledas del Sur, Guadalajara, Jalisco México. CP 44980.

(C) 2015 Revista de Psicoterapia 
Abstract
Objective: To identify the mental health of primary caregivers of children with brain injury and their impact on rehabilitation. Method: The primary caregivers of children with brain injury was assessed using the General Health Questionnaire (GHQ-28) validated for detecting psychological and psychiatric morbidity on Rehabilitation Center Telethon Child Occidente (Mexico). Cross-sectional study, was analyzed with nonparametric statistics. Results: We included 131 caregivers; $77 \%$ female, $23 \%$ male, average age 33 years, range 20-67. Biological parents were 95\% of caregivers. Caregivers showed more chronic altered mental health compared with the sharp 61.8 / 39.7\%, social dysfunction 45/3\%, anxiety / insomnia in the first indicator $40.5 / 12 \%$ somatic symptoms $29 / 7.6 \%$ and severe depression $9.2 / 2.3 \%(p<.001)$. The symptom most at risk for chronic psychiatric case was chronic anxiety and insomnia $O R=40.8$ ( $p$ <.001). The largest 12-month delay in the developmental age of the child was associated with acute mental health RM $3.8(p=.015)$ Parents with severe depression have high number of absences to therapies for their children $(p=.05)$. Conclusion: It is important to detect the risk of mental health disorders in primary caregiver, because of the direct negative effect on their welfare and their children.

Keywords: Anxiety and insomnia, primary caregiver, depression, social dysfunction, cerebral palsy, mental health. 


\section{Introducción}

Actualmente en México se reporta que la población infantil con discapacidad es de $1.9 \%$ la cual es adquirida durante el periodo perinatal en un $66.6 \%$, con alto porcentaje de dependencia funcional de un cuidador durante toda su vida (Instituto Nacional de Estadística y Geografía, 2011; Organización Mundial de la salud, 2011).

El impacto negativo ante un hijo recién nacido con deficiencias puede ocasionar trastornos en la salud psicosocial del cuidador principal e incrementa la prevalencia de morbilidad psiquiátrica comparado con cuidadores de niños sanos (Nuñez, 2007; Al-Kuwari, 2007). Las reacciones que presentan los cuidadores primarios son variables, desde un proceso de duelo con sentimientos teñidos de enojo, impotencia, desesperanza, confusión, rechazo, ansiedad, miedo, frustración, tristeza y culpa que conducen a la depresión (Muñoz-Lastra, 2011; Singer \& Frank, 2006). La responsabilidad del cuidador de un niño con discapacidad, tiene un efecto debilitante en el estado de ánimo con deterioro de su salud mental o estado de bienestar, consciente de sus propias capacidades, resolviendo situaciones adversas de la vida diaria, va desgastando el adecuado funcionamiento de los aspectos cognoscitivos, afectivos y conductuales (Achury, Castaño-Riaño, Gómez-Rubiano, \& Gueravara-Rodríguez, 2011; Organización Mundial de la Salud, 2007)

La naturaleza de la discapacidad, el débil estado de salud, la limitación funcional y la demanda requerida para el bienestar del niño son prioridad para el cuidador primario quién subestima su propia salud, encontrado una elevada sobrecarga en detrimento de su salud mental (Seguí, Ortiz-Tallo, \& De Diego, 2008). El continuo debilitamiento físico y mental de los cuidadores, es debido a la inadecuada adaptación y reestructuración de responsabilidades tanto en el proceso de rehabilitación como en el tratamiento especializado del hijo (Raina, y otros, 2005).

El impacto negativo ante la discapacidad de un hijo está relacionada con el estrés, ansiedad y preocupación por el futuro, la falta de descanso y tiempo para ellos mismos, con disminución de la energía física y psicosocial, que los va llevando a un deterioro crónico y progresivo de su salud que se va pasando por alto a través del tiempo (Murphy, Christian, Caplin, \& Young, 2007; Guevara-Benítez \& GonzálezSoto, 2012).

Debido a ello, este estudio tiene como objetivo la medición aguda y crónica de algunos indicadores de salud mental de los cuidadores en su mayoría padres que tienen la responsabilidad de cuidar a largo plazo de un hijo con discapacidad, desencadenando niveles altos de estrés y ansiedad, desgaste físico y emocional, lo cual tiene un efecto negativo directo en el bienestar de ellos y de sus hijos, comprometiendo la calidad de cuidado y apego al tratamiento requerido en el Centro de Rehabilitación Infantil Teletón de Occidente, de ahí la importancia de la prevención e intervención temprana que mitigue la cronificación y deterioro de la salud mental de los cuidadores primarios (Mahoney, Wiggers, Nam, Kralovic, \& 
Perales, 2014; Ritzema \& Sladeczek, 2011).

Para éste fin se utilizó el GHQ-28 que evalúa el estado de salud general, midiendo la angustia emocional y la incapacidad para seguir llevando a cabo funciones saludables normales y la aparición de algún malestar psíquico, desarrollado como una herramienta (screening) para detectar el riesgo a presentar desórdenes psiquiátricos, agudos y de larga duración. (Sterling, 2011).

\section{Material y métodos}

Participantes. Estudio de corte trasversal, no probabilístico, realizado en el Centro de Rehabilitación Infantil Teletón de Occidente ubicado en la ciudad de Guadalajara Jalisco (México), que otorga atención especializada e integral al paciente y su familia, con autorización de la Comisión de Ética en Investigación y previa firma de un consentimiento informado de los cuidadores de niños con diagnóstico de Parálisis cerebral de leve a severa de 1 a 5 años, sin distinción de género, seleccionados convenientemente según su asistencia a consulta de psicología clínica y atención familiar en el centro, en un periodo de Junio a diciembre de 2011.

Instrumentos y medidas. El GHQ-28 consta de 28 ítems agrupados en cuatro subescalas de 7 ítems cada una: subescala A (síntomas somáticos), subescala B (ansiedad e insomnio), subescala C (disfunción social) y subescala D (depresión grave). Cada pregunta tiene cuatro posibles respuestas tipo Likert. Para su calificación se utilizaron las puntuaciones GHQ número de síntomas agudos presentes asignando valores a las respuestas de $0,0,1,1 ;$ y CGHQ síntomas de larga duración, divide los ítems en dos apartados, los positivos que hacen referencia a la salud (A1,C1,C3,C4,C5,C6 y C7) puntúan 0,0,1,1 y el resto son negativos que hacen referencia a la enfermedad puntúan $0,1,1,1$. El punto de corte $5 / 6$, es indicativo de caso/no caso agudo y 12/13 de larga duración (Goldberg \& Williams, 1988; Goldberg, y otros, 1997). En ésta muestra la escala completa tuvo una confiabilidad de alfa de 0.85 .

El estado de desarrollo mental, capacidad global de inteligencia de los pacientes fue determinado con la Guía Portage que evalúa las habilidades por áreas de desarrollo desde el nacimiento a los 6 años, ésta inicialmente se creó para ofrecer servicios a los niños con discapacidad en comunidades rurales, emplea una evaluación sencilla para maestros y cuidadores. La tabla de observación y planificación a través de 5 áreas del desarrollo, Comunicación/Lenguaje/Lectoescritura, Desarrollo Socio-emocional, Exploración/Aproximaciones hacia el Aprendizaje, Actividad Motriz Intencional y Organización Sensorial. Se inicia la evaluación empleando el rango de edad por debajo de la edad cronológica, los rangos de edad, son: infantes (0-9, 9-18, 18-36 meses) y preescolar mayor a 36 meses. Esta tabla recoge la información sobre cada niño mediante la observación sistemática durante las actividades más usuales, con 3 posibilidades de respuesta siempre, ocasionalmente y no se puede observar. En la evaluación se avanza de edad si el niño cumple 
en el $75 \%$ de las habilidades y se finaliza si no domina por lo menos el $50 \%$ (Larson, $\mathrm{y}$ otros, 2006).

En aquellos niños de 4 a 5 años que tuvieron la capacidad, se les evaluó su habilidad global de inteligencia con la escala para preescolar WPPSI que se divide en dos sub escalas: Verbal (Información, Vocabulario, Aritmética, Semejanzas, Comprensión, Frases) y de Ejecución (Casa de los Animales, Figuras Incompletas, Laberintos, Diseños Geométricos, Diseño con Prismas y Claves), la sumatoria de las puntuaciones naturales se normalizaron para el promedio de cada edad (Wechsler, 1981).

Procedimiento. Se realizó la selección de dos grupos de pacientes con diagnóstico de Parálisis cerebral según la edad de 16 a 36 y 37 a 70 meses, atendidos integralmente (niño-cuidador) por un psicoterapeuta diferente cada grupo, quienes se encargaron de aplicar individualmente cara a cara a cada cuidador, el Goldberg's General Health Questionnaire-28 (GHQ-28). Además de evaluar a cada paciente el estado de desarrollo mental, capacidad global de inteligencia, según la edad se usaron dos diferentes escalas debido a que no fue posible evaluar el potencial intelectual del niño en sus primeros años porque muchas de la funciones no han aparecido o continúan desarrollándose, por ejemplo; el lenguaje, coordinación visomotora, etc.

Se obtuvieron datos sociodemográficos de los cuidadores: edad, género, parentesco, localización de la vivienda dentro o fuera de la ciudad (local o foráneo respectivamente), tipo de familia (nuclear, extensa o uniparental), apoyo económico (subsidio o costo de la rehabilitación dentro de la institución).

Análisis estadístico. Se usó estadística no paramétrica. Se describieron las frecuencias de las características del cuidador y de los pacientes, según escalas de medición de las variables, usando media, desviación estándar y proporciones, se compararon las proporciones de caso psiquiátrico agudo y crónico como variable dependiente dicotómica y cada una de los estratos de las variables independientes tanto del paciente como del cuidador, mediante $\mathrm{X}^{2}$ exacto de Fisher o McNemar. Se midió la asociación entre variables, síntomas de salud mental aguda y crónica mediante análisis bivariado. La fuerza y sentido de la asociación se determinó mediante el cálculo de razón de momios con su respectivo intervalo de confianza de 95\%. Se usaron los programas estadísticos SPSS 19 y PRIMER.

\section{Resultados}

Se incluyeron 131 cuidadores; 77\% femeninos, edad promedio 33 años, fueron $95 \%$ padres biológicos, el tiempo promedio al cuidado del hijo discapacitado fue 45 meses y el de acudir a atención integral niño/cuidador en el centro casi dos años. La variabilidad de las características sociodemográfias no modificó el estado de salud mental del cuidador (Tabla 1). 
Tabla 1.

Frecuencias de características de cuidador primario y salud mental

\begin{tabular}{|c|c|c|c|c|c|}
\hline & $\begin{array}{c}\text { Frecuencia } \\
(\%) \\
\text { N } 131\end{array}$ & $\begin{array}{l}\mathrm{GHQ} \\
\text { Caso }\end{array}$ & $\begin{array}{r}\text { Sig. exacta } \\
\text { (unilateral) }\end{array}$ & $\begin{array}{l}\text { CGHQ } \\
\text { Caso }\end{array}$ & $\begin{array}{r}\text { Sig. exacta } \\
\text { (unilateral) }\end{array}$ \\
\hline \multicolumn{6}{|c|}{ Edad promedio años $33 \pm 8.87$ (20 a 67) } \\
\hline$<25$ & $26(19.8)$ & 13 & 0.443 & 19 & 0.125 \\
\hline $25-35$ & $59(45)$ & 23 & & 31 & \\
\hline$>35$ & $46(35.2)$ & 16 & & 31 & \\
\hline \multicolumn{6}{|l|}{ Género \% } \\
\hline Femenino & $101(77)$ & 44 & 0.072 & 63 & 0.488 \\
\hline Masculino & $30(23)$ & 8 & & 18 & \\
\hline \multicolumn{6}{|c|}{ Parentesco del cuidador primario \% } \\
\hline Madre & $96(73)$ & 42 & 0.190 & 60 & 0.857 \\
\hline Padre & $29(22)$ & 8 & & 17 & \\
\hline Abuela & $5(4)$ & 1 & & 3 & \\
\hline Abuelo & $1(1)$ & 1 & & 1 & \\
\hline \multicolumn{6}{|l|}{ Localización vivienda \% } \\
\hline Local & $98(75)$ & 38 & 0.432 & 59 & 0.328 \\
\hline Foráneo & $33(25)$ & 14 & & 22 & \\
\hline \multicolumn{6}{|l|}{ Tipo de familia } \\
\hline Nuclear & $90(69)$ & 35 & 0.956 & 58 & 0.632 \\
\hline Extensa & $31(24)$ & 13 & & 17 & \\
\hline Uniparental & $10(7)$ & 4 & & 6 & \\
\hline \multicolumn{6}{|c|}{ Apoyo Económico en rehabilitación \% } \\
\hline 100 & $4(3)$ & 3 & 0.271 & 1 & 0.305 \\
\hline 90 & $119(91)$ & 45 & & 75 & \\
\hline 80 & $8(6)$ & 4 & & 5 & \\
\hline \multicolumn{6}{|c|}{ Asistencia al CRIT años promedio 1.85 $\pm 1.1(1-5)$} \\
\hline
\end{tabular}

* Estadísticamente significativo (0.05) Chi-cuadrado, (exacto de Fisher/McNemar)

Los pacientes fueron 63\% hombres, edad promedio 3.7 años, el diagnóstico realizado por el médico rehabilitador fue lesión cerebral, 15.6\% severa, según GMFCS (Gross Motor Function Classificaction System). E1 77\% de los pacientes tenían más de 12 meses de retardo en edad madurativa evaluado con Guía Portage quienes tuvieron en mayor proporción alteración de la salud mental aguda y crónica. Con riesgo de ser caso agudo RM 3.8 el Intervalo de Confianza (IC) 1.18-12.4 $(\mathrm{p}=0.015)$ y de ser caso crónico RM 2.9, IC de 0.99-6.9 $(\mathrm{p}=0.08)$ debido a que aun sin estar expuestos al retardo de edad madurativa del niño $>12$ meses, se cronificaron en un $50 \%$ (Tabla 2). 
Tabla 2.

Frecuencias de las características del niño con parálisis cerebral y salud mental del cuidador

\begin{tabular}{|c|c|c|c|c|c|}
\hline & $\begin{array}{c}\text { Frecuencia } \\
(\%) \\
N 131\end{array}$ & $\begin{array}{l}\text { GHQ } \\
\text { Caso }\end{array}$ & $\begin{array}{r}\text { Sig. exacta } \\
\text { (unilateral) }\end{array}$ & $\begin{array}{c}\text { CGHQ } \\
\text { Caso }\end{array}$ & $\begin{array}{r}\text { Sig. exacta } \\
\text { (unilateral) }\end{array}$ \\
\hline \multicolumn{6}{|c|}{ Edad años promedio meses $45.5 \pm 13.5$ (18-71) } \\
\hline$<3$ años & $39(30)$ & 11 & \multirow[t]{2}{*}{.059} & 25 & \multirow[t]{2}{*}{0.442} \\
\hline >3 años & $92(70)$ & 41 & & 56 & \\
\hline \multicolumn{6}{|l|}{ Género \% } \\
\hline Masculino & $82(62.6)$ & 34 & \multirow[t]{2}{*}{.364} & 53 & \multirow[t]{2}{*}{0.251} \\
\hline Femenino & $49(37.4)$ & 18 & & 28 & \\
\hline \multicolumn{6}{|c|}{ Cociente intelectual promedio $82.5 \pm 19(48-111)$} \\
\hline $\mathrm{Cl}$ Mayor de 70 & $21(65)$ & 9 & .513 & 10 & \multirow[t]{2}{*}{0.410} \\
\hline Cl Menor de 70 & $11(35)$ & 4 & & 4 & \\
\hline \multicolumn{6}{|c|}{ Retardo en edad madurativa meses promedio $20.4 \pm 9.4(2-40)$} \\
\hline Retardo mayor & & & & & \multirow{3}{*}{$0.045^{*}$} \\
\hline $\begin{array}{l}\text { de } 12 \text { meses } \\
\text { Retardo menor }\end{array}$ & $76(77.5)$ & 35 & $0.01^{*}$ & 55 & \\
\hline de 12 meses & $22(22.5)$ & 4 & & 11 & \\
\hline \multicolumn{6}{|c|}{ Retardo en aproximaciones hacia el aprendizaje meses promedio $18 \pm 11(3-55)$} \\
\hline $\begin{array}{l}\text { Retardo mayor } \\
\text { de } 12 \text { meses }\end{array}$ & $64(65.3)$ & 27 & .329 & 46 & \multirow[t]{3}{*}{0.139} \\
\hline Retardo menor & & & & & \\
\hline de 12 meses & $34(34.7)$ & 12 & & 20 & \\
\hline \multicolumn{6}{|c|}{ Retardo en desarrollo socioemocional meses promedio $20.4 \pm 9.4(2-40)$} \\
\hline $\begin{array}{l}\text { Retardo mayor } \\
\text { de } 12 \text { meses }\end{array}$ & $60(61)$ & 25 & .397 & 43 & \multirow[t]{3}{*}{0.177} \\
\hline Retardo menor & & & & & \\
\hline de 12 meses & $38(39)$ & 14 & & 23 & \\
\hline \multicolumn{6}{|l|}{ Severidad de discapacidad \% } \\
\hline Leve & $28(21.4)$ & 8 & 0.172 & 18 & \multirow[t]{3}{*}{0.634} \\
\hline Moderada & $83(63.3)$ & 38 & & 49 & \\
\hline Severa & $20(15.3)$ & 6 & & 14 & \\
\hline \multicolumn{6}{|l|}{ Grado de discapacidad \% } \\
\hline Diparesia & $63(48)$ & 27 & .597 & 38 & \multirow[t]{3}{*}{0.304} \\
\hline Triparesia & $8(6)$ & 2 & & 7 & \\
\hline Cuadriparesia & $60(46)$ & 23 & & 36 & \\
\hline
\end{tabular}

* Estadísticamente significativo (0.05) Chi-cuadrado, (exacto de Fisher/McNemar)

Los síntomas agudos se manifestaron en menor proporción que los crónicos: Los somáticos 7.6/ 29, ansiedad/insomnio 12.2/40.4, disfunción social 3/45, depresión 2.2/9.1 respectivamente; de estos la depresión evolucionó a la cronificación en $93 \%$ y la ansiedad e insomnio en $75 \%$. La disfunción social casi no es percibida en forma aguda pero si en la crónica (Tabla 3). 
Tabla 3.

Análisis bivariado, asociación de Cronificación de síntomas de salud mental en el cuidador primario

\begin{tabular}{|c|c|c|c|c|c|c|c|c|c|}
\hline & & & & $X^{2}$ & Sig. & $\mathrm{RM}$ & $\begin{array}{c}\text { IC } \\
\text { menor }\end{array}$ & $\begin{array}{c}\text { IC } \\
\text { mayor }\end{array}$ & Sig. \\
\hline & & $\begin{array}{l}\text { toma } \\
\text { cró }\end{array}$ & $\begin{array}{l}\text { omá } \\
\text { cos }\end{array}$ & ticos & & & & & \\
\hline & & No & Sí & & & & & & \\
\hline \multirow{4}{*}{$\begin{array}{l}\text { Síntomas somáticos } \\
\text { agudos }\end{array}$} & No & 90 & $\begin{array}{r}31 \\
7\end{array}$ & 8.83 & 0.006 & 0.37 & 0.22 & 0.61 & $0.003^{*}$ \\
\hline & Sí & 3 & 7 & & & & & & \\
\hline & & $\begin{array}{r}\text { sieda } \\
\mathrm{cr}\end{array}$ & $\begin{array}{l}\text { nson } \\
\text { co }\end{array}$ & nnio & & & & & \\
\hline & & No & Sí & & & & & & \\
\hline \multirow{4}{*}{$\begin{array}{l}\text { Ansiedad/insomnio } \\
\text { agudo }\end{array}$} & No & 74 & 41 & 9.03 & 0.003 & 2.57 & 1.09 & 6.08 & $0.003^{*}$ \\
\hline & Sí & 4 & 12 & & & & & & \\
\hline & & $\begin{array}{r}\text { isfunc } \\
\mathrm{cr}\end{array}$ & $\begin{array}{l}\text { n so } \\
\text { ca }\end{array}$ & cial & & & & & \\
\hline & & No & Sí & & & & & & \\
\hline \multirow{4}{*}{$\begin{array}{l}\text { Disfunción social } \\
\text { aguda }\end{array}$} & No & 70 & 57 & 0.04 & 0.612 & 0.90 & 0.33 & 2.44 & 0.839 \\
\hline & Sí & 2 & 2 & & & & & & \\
\hline & & Dep & sión & & & & & & \\
\hline & & No & Sí & & & & & & \\
\hline Depresión & No & 119 & 9 & 30.45 & 0.001 & 0.07 & 0.04 & 0.13 & $<.001$ \\
\hline aguda & Sí & 0 & 3 & & & & & & \\
\hline
\end{tabular}

* Estadísticamente significativo (0.05) Chi-cuadrado, exacto de Fisher

El CGHQ-28 detectó que casi dos terceras partes de los cuidadores manifiestan alteración en su salud mental crónica, a diferencia del poco más de una tercera parte de los agudos. Los que presentan síntomas somáticos, ansiedad e insomnio de larga duración tuvieron riesgos elevados de ser caso psiquiátrico crónico con RM 40-53 (Tabla 4). 
Tabla 4.

Análisis bivariado, asociación de alteración de salud mental y síntomas afectivos en padres de niños con parálisis cerebral

\begin{tabular}{lrrrrrrrrr}
\hline & $\begin{array}{r}\text { No caso } \\
\text { Crónico }\end{array}$ & $\begin{array}{c}\text { Caso } \\
\text { Crónico }\end{array}$ & $X^{2}$ & P & RM & $\begin{array}{c}\text { IC } \\
\text { menor }\end{array}$ & $\begin{array}{c}\text { IC } \\
\text { mayor }\end{array}$ & P \\
\hline Caso psiquiátrico agudo & & & & & & & & & \\
No & 44 & 35 & & & & & & \\
Si & 6 & 46 & 25.9 & $<.001$ & 9.64 & 3.69 & 25.16 & $<.001^{*}$ \\
Síntomas somáticos crónicos & & & & & & & & \\
No & 50 & 43 & & & & & & \\
Si & 0 & 38 & 30.8 & $<.001$ & 0.46 & 0.37 & 0.58 & $<.001^{*}$ \\
Ansiedad/insomnio agudo & & & & & & & & & \\
No & 48 & 67 & & & & & & \\
Si & 2 & 14 & 3.92 & 0.02 & 5.01 & 1.09 & 23.1 & $0.05^{*}$ \\
Ansiedad/insomnio crónicos & & & & & & & & \\
No & 48 & 30 & & & & & & \\
Si & 2 & 51 & 42.2 & $<.001$ & 40.8 & 9.24 & 180.1 & $<.001^{*}$ \\
Disfunción social crónica & & & & & & & & & \\
No & 41 & 31 & & & & & & \\
Si & 9 & 50 & 22.15 & $<.001$ & 7.35 & 3.14 & 17.18 & $<.001^{*}$ \\
Depresión crónica & & & & & & & & & \\
No & 49 & 70 & & & & & & \\
Si & 1 & 11 & 4.98 & 0.03 & 7.7 & 0.96 & 61.6 & 0.06 \\
\hline
\end{tabular}

* Estadísticamente significativo (0.05) Chi-cuadrado, Mantel-Haenszel

Se detectó mayor número de inasistencias en los padres que presentaron depresión crónica $83 / 17 \%$ con $\mathrm{p}=0.05$.

\section{Discusión}

Diversas investigaciones han demostrado que la salud mental del cuidador primario que atiende a niños con alguna discapacidad, sufren fatiga, depresión, insomnio, culpa, baja estima, ansiedad y otros síntomas somáticos asociados al estrés reflejados en los problemas de salud física (Seguí, Ortiz-Tallo, \& De Diego, 2008; Pozo-Cabanillas, Sarriá-Sánchez, \& Méndez-Zaballos, 2006; Gallagher \& Whiteley, 2013).

En éste trabajo se encontró que en su mayoría los cuidadores primarios de niños con parálisis cerebral mostraron alteración de su salud mental cronificada, duplicando proporcionalmente los síntomas agudos, éste estado se vuelve un estilo de vida, con deterioro general debido a que no logran transformar la discapacidad de su hijo en una realidad, los padres presentan una adaptación más que una resolución o recuperación. Lo anterior constituye un patrón conductual, con una serie de disfunciones fisiológicas y trastornos del estado de ánimo de larga duración (Ha, Greenberg, \& Seltzer, 2011).

Desde el nacimiento de su hijo ellos sufren duelos ocultos, pues no son reconocidos ni validados públicamente (Zambrano-Cruz \& Ceballos-Cardona, 
2007; Bemister, Brooks, Dyck, \& Kirto, 2014). Por lo tanto, los procesos no resueltos, se van agravando con la rutina de la rehabilitación integral, dando prioridad a la atención del hijo con discapacidad, posponiendo o desatendiendo sus propias necesidades en detrimento de su salud mental comparada con los padres de niños sin discapacidad (Lach, y otros, 2009).

Los síntomas somáticos asociados al estrés se reflejaron principalmente como dolor de cabeza y agotamiento sin tener un diagnóstico de enfermedad física (Brehaut, y otros, 2004).

El insomnio o ausencia de un sueño reparador se manifestó desde la fase aguda y a lo largo del cuidado del paciente causando humor alterado, tensión, ansiedad y desinterés en la rehabilitación integral de su hijo, reflejado en un ánimo deprimido relacionado con inasistencias en el proceso de tratamiento en el Centro con incumplimiento del reglamento institucional (Marx, Masruha, Masruha, \& Vilanova, 2011).

La disfunción social descrita como incapacidad de disfrutar las actividades diarias, insatisfacción y pobre gratificación en el desempeño de la actividad como cuidador no se percibe en la fase aguda pero alcanzó el valor más alto en la etapa crónica (Cheshire, Barlow, \& Powell, 2010).

El síntoma más grave encontrado fue la depresión, con indicadores como desvalorización, desesperanza e ideación suicida (Zambrano-Cruz \& CeballosCardona, 2007; Magaña \& Smith, 2006; De Alba, Castellanos, \& Sánchez, 2015).

Los cuidadores primarios continúan enfrentándose con la discapacidad en la misma rutina, han agotado sus recursos de aprendizaje, por lo tanto se van limitando y perturbando por los intentos en busca del bienestar de su hijo, sin darse cuenta, que van desgastándose a lo largo del tiempo. En dicho periodo de tiempo, no han atendido a sus necesidades personales y sociales. Ellos requieren mayores redes de apoyo además de la familia, profesionales que intervengan clínica y psicoterapéuticamente en su salud mental (Martínez, Robles, Ramos, Santiesteban, \& García, 2008; Thrush \& Hyder, 2014).

\section{Conclusiones}

Estos indicadores son significativos en el estudio, por que identifican que el cuidador primario desarrolla alto riesgo de presentar desórdenes psiquiátricos, agudos y de larga duración, por centrar la atención en el hijo con discapacidad en los primeros años de rehabilitación La angustia emocional lo incapacitan para seguir llevando a cabo funciones saludables normales.

\section{Limitaciones del estudio}

Este estudio tiene varias limitaciones que están intrínsecamente asociadas con el instrumento de medición GHQ-28, No realiza un diagnóstico pero sí detecta trastornos psicológicos en ambientes no psiquiátricos.

Un sesgo de selección a nuestros hallazgos es que no pueden ser generalizables 
a todos los cuidadores de niños con discapacidad, aún dentro del mismo centro nuestra muestra no fue representativa, además el modelo de tratamiento solicita a los tutores legales o padres que asistan y sean responsables del cuidado y rehabilitación del niño, por eso nuestra población en su mayoría son padres biológicos, tampoco se evaluaron controles.

La entrevista individual podría sesgarse debido a que no se mantiene el anonimato y seguridad psicológica, ya que la entrevista es familiar. Otro factor que limita la generalización de los resultados es que no todos los cuidadores están recibiendo apoyo oportuno y permanente durante el proceso de rehabilitación.

A pesar de estas limitaciones, creemos que los resultados de este estudio tiene implicaciones interesantes sobre cómo síntomas de larga duración influyen en la alteración de la salud mental crónica, independientemente del contexto, sin que por ello los cuidadores soliciten ayuda psiquiátrica, o un relevo en el cuidado de su paciente a largo plazo, que puedan sostenerlos en los roles de cuidado.

\section{Referencias bibliográficas}

Achury, D. M., Castaño-Riaño, H. M., Gómez-Rubiano, L. M. y Gueravara-Rodríguez, N. M. (2011). Calidad de vida de los cuidadores de pacientes con enfermedades crónicas con parcial dependencia. Investigación en Enfermería: Imagen y Desarrollo, 13(1).

Al-Kuwari, M. (2007). Psychological health of mothers caring for mentally disabled children in Qatar. Neurosciences, 2(4), 312-317.

Bemister, T. B., Brooks, B. L., Dyck, R. H. y Kirto, A. (2014). Parent and family impact of raising a child with perinatal stroke. BMC Pediatr, 14, 182.

Brehaut, J. C., Kohen, D. E., Raina, P., Walter, S. D., Russell, D. J., Swinton, M., . . Rosenbaum, P. (2004). The Health of Primary Caregivers of Children With Cerebral Palsy: How Does It Compare With That of Other Canadian Caregivers? Pediatrics, 114(2), 182-191.

Cheshire, A., Barlow, J. H. y Powell, L. A. (2010). The psychosocial well-being of parents of children with cerebral palsy: a comparison study. Disability and Rehabilitation, 32(20), 1673-1677.

De Alba, M., Castellanos, A. y Sánchez, L. M. (2015). Riesgo Suicida y Síntomas Depresivos en Padres de Hijos con Enfermedad Neuromuscular. Acta de Investigación Psicológica, 5(1), 1872-1880.

Gallagher, S. y Whiteley, J. (2013). The association between stress and physical health problems in parents caring for children with intellectual disabilities is moderated by children's challenging behaviours. Journal of health psychology, 18(9), 1220-31.

Goldberg, D. y Williams, P. (1988). A User's Guide to the GHQ. (NFER-Nelson, Ed.) Windsor, England: Publishing.

Goldberg, D., Gater, R., Sartorius, N., Ustun, T., Piccinelli, M., Gureje, O. y Rutter, C. (1997). The validity of two versions of the GHQ in the WHO study of mental health. Psychological Medicine, 27, 191-197.

Guevara-Benítez, Y. y González-Soto, E. (2012). Las familias ante la discapacidad. Revista Electrónica de Psicología Iztacala, 15(3), 1023-1050.

Ha, J. H., Greenberg, J. S. y Seltzer, M. M. (2011). Parenting a Child With a Disability: The Role of Social Support for African American Parents. The Journal of Contemporary Social Services, 92(4), 405-411.

Instituto Nacional de Estadística y Geografía. (2011). Resultados definitivos del Censo de Población y Vivienda 2010 .

Lach, L. M., Kohen, D. E., Garner, R. E., Brehaut, J. C., Miller, A. R., Klassen, A. F. y Rosenbaum, P. L. (2009). The health and psychosocial functioning of caregivers of children with neurodevelopmental disorders. Disability and Rehabilitation, 31(8), 607-618. 
Larson, N., Herwig, J., Wollenburg, K., Olsen, E., Bowe, W., Chvojicek, R. y Copa, A. (2006). Guía Portage Nacimiento a Seis (Primera edición en español ed.). (W. Portage, Ed.) Wisconsin, Estados Unidos: Cooperative Educational Service Agency 5.

Lewis , J. E., Feaster, D. J., Czaja, S. J., Eisdorfer, C., Shculz, R. y Szapocznik, J. (2006). The role of family functioning in the stress process of dementia caregivers: a structural family framework. Gerontologist, $46(1)$.

Madrigal-Muñoz, A. (2007). Familias ante la parálisis cerebral. Intervención Psicosocial, 16(1).

Magaña, S. y Smith, M. J. (2006). Health Outcomes of Midlife and Older Latina and Black American Mothers of Children With Developmental Disabilities. Mental Retardation, 44(3), 224-234.

Mahoney, G., Wiggers, B., Nam, S., Kralovic, S. y Perales, F. (2014). How Depressive Symptomatology of Mothers of Children with Pervasive Developmental Disorders Relates to their Participation in Relationship Focused Intervention. International Journal of Early Childhood Special Education (INT-JECSE), 6(2), 204-221.

Martínez, L., Robles, M. T., Ramos, B., Santiesteban, F. y García, M. E. (2008). Carga percibida del cuidador primario del paciente con parálisis cerebral infantil severa del Centro de Rehabilitación Infantil Teletón. Revista Mexicana de Medicina Física y Rehabilitación(20).

Marx, C., Masruha, R. E., Masruha, R. M. y Vilanova, L. C. (2011). Depression, anxiety and daytime sleepiness of primary caregivers of children with cerebral palsy. Revista Paulista de Pediatria, 29(4), 483-488.

Muñoz-Lastra, A. (2011). Impacto de la discapacidad en las familias. Revista Digital Inovación y Experiencias Educativas(38), 1-8.

Murphy, N. A., Christian, B., Caplin, D. A. y Young, P. C. (2007). The health of caregivers for children with disabilities: caregiver perspectives. Child Care Health Dev, 33(2), 180-187.

Nuñez, B. (2007). Familia y discapacidad: de la vida cotidiana a la teoría. Buenos Aires: Lugar Editorial.

Oramas-Viera, A., Santana-López, S. y Vergara-Barrennecha, A. (2006). El bienestar psicológico, un indicador positivo de la salud mental. Revista Cubana de Salud y Trabajo, 7(1), 34-39.

Organización Mundial de la Salud. (2007). Recuperado de: http://www.who.int/features/qa/62/es/

Organización Mundial de la salud. (2011). Clasificación Internacional del Funcionamiento, de la Discapacidad y de la Salud(Segunda ed.).(C. d. publicaciones, Ed.) Madrid, España: Ministerio de sanidad, politica social e igualdad.

Organización Mundial de la Salud. (2011). Resumen Informe Mundial sobre la discapacidad. Ginebra.

Pozo-Cabanillas, P., Sarriá-Sánchez, E. y Méndez-Zaballos, L. (2006). Estrés en madres de personas con trastornos del espectro autista. Psicothema, 18(3), 342-347.

Raina, P., O’Donell, M., Rosenbaum, P., Brehaut, J., Walter, S. D. y Russell, S. (2005). The health and well-being of caregivers of children with cerebral palsy. Pediatrics, 115(6), 626-636.

Ritzema, A. M. y Sladeczek, I. E. (2011). Stress in Parents of Children with developmental disabilities over Time. Journal on Developmental Disabilities, 17( 2), 21-34.

Rodrigo-López, M. J. (2009). Una mirada integradora de la resiliencia parental: desde el contexto hasta la mente de las madres y los padres en riesgo psicosocial. Psic. da Ed., 28, 51-71.

Seguí, J. D., Ortiz-Tallo, M. y De Diego, Y. (2008). Factores asociados al estrés del cuidador primario de niños con autismo: Sobrecarga, psicopatología y estado de salud. Anales de psicología, 24(1), 100-105.

Singer, G. H. y Frank, F. (2006). Meta-Analysis of Comparative Studies of Depression in Mothers of Children With and Without Developmental Disabilities. American Journal on Mental Retardation:, 111(3), 155-169.

Sterling, M. (2011). General Health Questionnaire - 28 (GHQ-28). Journal of Physiotherapy, 57(4), 259.

Thrush, A. y Hyder, A. (2014). The neglected burden of caregiving in low- and middle-income countries. Disability and Health Journal, 7, 262-272.

Wechsler, D. (1981). Wppsi-Español Escala de inteligencia para los niveles preescolar y primario. DF, México: El manual moderno.

Zambrano-Cruz, R. y Ceballos-Cardona, P. (2007). Síndrome de carga del cuidador. Revista Colombiana de Psiquiatría, 36(1). 